\title{
Favorable Bridging Therapy Based on DWI-FLAIR Mismatch in Patients with Unclear-Onset Stroke
}

\author{
(D). Mourand, D. Milhaud, (DC. Arquizan, (D). Lobotesis, R. Schaub, (D). Machi, (DX. Ayrignac, (D).F. Eker, (D)A. Bonafé,
} and (D). Costalat

\begin{abstract}
BACKGROUND AND PURPOSE: Standard selection criteria for revascularization therapy usually exclude patients with unclear-onset stroke. Our aim was to evaluate the efficacy and safety of revascularization therapy in patients with unclear-onset stroke in the anterior circulation and to identify the predictive factors for favorable clinical outcome.
\end{abstract}

MATERIALS AND METHODS: We retrospectively analyzed 41 consecutive patients presenting with acute stroke with unknown time of onset treated by intravenous thrombolysis and/or mechanical thrombectomy. Only patients without well-developed fluid-attenuated inversion recovery changes of acute diffusion lesions on MR imaging were enrolled. Twenty-one patients were treated by intravenous thrombolysis; 19 received, simultaneously, intravenous thrombolysis and mechanical thrombectomy (as a bridging therapy); and 1 patient, endovascular therapy alone. Clinical outcome was evaluated at 90 days by using the mRS. Mortality and symptomatic intracranial hemorrhage were also reported.

RESULTS: Median patient age was 72 years (range, 17-89 years). Mean initial NIHSS score was $14.5 \pm 5.7$. Successful recanalization (TICI 2b-3) was assessed in $61 \%$ of patients presenting with an arterial occlusion, symptomatic intracranial hemorrhage occurred in 2 patients (4.9\%), and 3 (7.3\%) patients died. After 90 days, favorable outcome (mRS $0-2$ ) was observed in 25 (61\%) patients. Following multivariate analysis, initial NIHSS score (OR, 1.43; 95\% Cl, 1.13-1.82; $P=.003)$ and bridging therapy (OR, 37.92; 95\% Cl, 2.43-591.35; $P=.009)$ were independently associated with a favorable outcome at 3 months.

CONCLUSIONS: The study demonstrates the safety and good clinical outcome of acute recanalization therapy in patients with acute stroke in the anterior circulation and an unknown time of onset and a DWI/FLAIR mismatch on imaging. Moreover, bridging therapy versus intravenous thrombolysis alone was independently associated with favorable outcome at 3 months.

ABBREVIATIONS: FAT = first found abnormal time; GRAPPA = generalized autocalibrating partially parallel acquisition; IVT = intravenous thrombolysis; sICH = symptomatic intracranial hemorrhage

A cute ischemic strokes with an unknown time of symptom onset occur in approximately $25 \%$ of patients. ${ }^{1}$ Hence, these patients are usually excluded from intravenous thrombolysis (IVT). ${ }^{2}$ However, many patients with an unknown stroke onset could also benefit from this treatment. In a subset of these patients, it has been shown that the clinical features and imaging characteristics do not differ significantly from those in patients

Received January 6, 2015; accepted after revision June 2

From Departments of Neurology (I.M., D.M., C.A., X.A.), Neuroradiology (K.L., P.M., O.F.E., A.B., V.C.), and Medical Information (R.S.), University Hospital Center of Montpellier, Gui de Chauliac Hospital, Montpellier, France.

A.B. and V.C. contributed equally to this work.

Please address correspondence to I. Mourand, MD, Neurology Department, CHRU, Gui de Chauliac Hospital, 80 Avenue Augustin Fliche, 34295 Montpellier Cedex 5,

France; e-mail: i-mourand@chu-montpellier.fr

三 Indicates article with supplemental on-line table.

http://dx.doi.org/10.3174/ajnr.A4574 with a known time of onset. ${ }^{1} \mathrm{MR}$ imaging could be helpful if it is used as a "clock" for stroke of unknown time onset; indeed, in a recent multicenter observational study of patients with stroke with known time of symptom onset, the DWI-FLAIR mismatch, defined by positive findings on DWI and negative findings on FLAIR, was effective in identifying patients within 4.5 hours of symptom onset. ${ }^{3}$

To date, limited studies have focused on the safety and effectiveness of IVT in patients with a stroke of unknown onset time, especially by using MR imaging-specific eligibility criteria. ${ }^{4-9}$ Only a few reports have evaluated the feasibility of endovascular therapies in patients with wake-up stroke. ${ }^{10-12}$ Recently, randomized studies have demonstrated that mechanical thrombectomy is an alternative and synergistic method of treatment to IVT in acute ischemic stroke, with a higher recanalization rate $(66 \%-100 \%)$ and a more favorable outcome $(32.6 \%-71 \%) .^{13-17}$

The aim of this study was to describe the experience of our 
center with 41 patients presenting with a stroke of an unknown time of symptom onset in the anterior circulation, who were treated by using a coalescent stroke-management protocol with IVT, mechanical thrombectomy, or bridging therapy based on DWI/FLAIR mismatch. We also assessed predictive factors for favorable outcome at 3 months and evaluated the feasibility, safety, and efficacy of revascularization therapy in these patients.

\section{MATERIALS AND METHODS}

All consecutive patients admitted in our stroke unit with a stroke of unknown time of onset and treated by reperfusion therapy between October 2010 and October 2013 were included in this study. The patient demographics, risk factors, and clinical and imaging data were prospectively registered in our stroke databank. The NIHSS score was systematically assessed by a stroke neurologist on admission. This study was approved by the local ethics committee. Consent for treatment was obtained from the patients or their family before the endovascular procedure.

The inclusion criteria were as follows: 1) patients with acute stroke without a known time of symptom onset, presenting to our emergency department within 4 hours after the first found abnormal time (FAT); 2) acute ischemic lesions within the anterior circulation on DWI; 3) an ASPECTS of $\geq 5$; 4) the presence of a DWI/FLAIR mismatch according to Thomalla et $\mathrm{al}^{3}$; 5) the presence of a clinically relevant impairment in social, occupational, or other important areas of functioning following a physician evaluation; and 6) the presence of clinical-diffusion mismatch between stroke severity and volume of DWI lesions assessed by visual inspection. For mechanical thrombectomy, patients were selected if they had the following additional criteria: 1) the presence of a proximal intracranial artery occlusion in the stroke territory; 2) an NIHSS score of $\geq 8$; 3 ) a premorbid $\mathrm{mRS}$ of $<1$; and 4) initiation of endovascular treatment within 6 hours of FAT.

In cases of anterior cerebral artery stroke, the MR imaging eligibility criterion was an infarct volume $<50 \%$ of the arterial territory.

\section{MR Imaging Protocol}

Multimodal MR imaging was performed in all patients by using a 1.5T magnet (Gyroscan Intera, Release 10; Philips Healthcare, Best, the Netherlands; 33-mT/m hypergradients). The MR imaging protocol included the following sequences: $\mathrm{T} 2$ gradient echo $\left(\mathrm{TR} / \mathrm{TE}=900 / 27 \mathrm{~ms}\right.$; flip angle $=15^{\circ}$; 1 repetition; generalized autocalibrating partially parallel acquisition [GRAPPA] $=2$; $5.0-\mathrm{mm}$ section thickness with no intersection gap; voxel size $=$ $1.3 \times 0.9 \times 5 \mathrm{~mm}) ; \mathrm{DWI}(\mathrm{TR} / \mathrm{TE}=3600 / 83 \mathrm{~ms}$; b-values $=0$ and $1000 \mathrm{~s} / \mathrm{mm}^{2} ; 2$ repetitions; GRAPPA $=2 ; 5.0-\mathrm{mm}$ section thickness with no intersection gap; voxel size $=1.8 \times 1.8 \times 5 \mathrm{~mm})$; an ADC map; FLAIR $(\mathrm{TR} / \mathrm{TE}=8000 / 94 \mathrm{~ms}$; $\mathrm{TI}=2500 \mathrm{~ms}$; turbo factor $=15$; GRAPPA $=2 ; 5.0-\mathrm{mm}$ section thickness with no intersection gap; voxel size $=0.6 \times 0.6 \times 5 \mathrm{~mm}$ ); and $\mathrm{T} 1$ contrast-enhanced MRA (3D coronal gradient echo: TR/TE $=3.45$ / $1.28 \mathrm{~ms}$; flip angle $=25^{\circ}$; GRAPPA $=2 ; 144$ sections; voxel size $=$ $0.7 \times 0.7 \times 0.7 \mathrm{~mm}$; gadolinium contrast agent, $0.5 \mathrm{mmol} / \mathrm{mL}$, $0.2 \mathrm{~mL} / \mathrm{kg}$; flow rate $=2 \mathrm{~mL} / \mathrm{sec}$ ) of the supra-aortic trunks and intracranial vessels.

The DWI/FLAIR mismatch was defined according to Thom- alla et al. $^{3}$ It was diagnosed when a visible acute ischemic lesion was present on DWI with no traceable parenchymal hyperintensity in the corresponding region on FLAIR imaging. Collateral blood flow in the distal cerebral artery territory was defined on FLAIR by linear or serpentine vascular hyperintensities relative to gray matter in the MCA territory subarachnoid space. It was graded as "present" if vascular hyperintensities in the sulci were seen on FLAIR images or as "absent" if they were not detectable.

\section{Revascularization Protocol}

IVT $(0.9 \mathrm{mg} / \mathrm{kg})$ was administered to patients within a maximum of 4.5 hours of FAT. Conventional clinical and laboratory inclusion and exclusion criteria for IVT were applied. ${ }^{2}$ In cases of bridging therapy, patients were transferred to the angiographic suite for thrombectomy as soon as possible. Among patients with contraindications to IVT, thrombectomy alone was performed.

Mechanical thrombectomy was performed via a femoral artery approach with the patient under general anesthesia with the Solitaire FR device (Covidien, Irvine, California). General anesthesia included urinary bladder catheterization and endotracheal intubation without neuromuscular blockade.

An 8 F or 9F Merci balloon-guide catheter (Concentric Medical, Mountain View, California) was inserted through a sheath. A 0.21-inch-internal-diameter microcatheter (Prowler Select Plus; Codman \& Shurtleff, Raynham, Massachusetts; or Vasco 21; Balt, Montmorency, France) was navigated distal to the occlusion over a 0.014 -inch steerable guidewire, which was then exchanged with the thrombectomy device. During the retrieval, the balloon-guide catheter was inflated to interrupt anterograde flow. Manual aspiration with a $50-\mathrm{mL}$ syringe was performed through the hemostatic valve during the retrieval, to reverse the flow and aspirate clot debris possibly lost in the guide catheter lumen. The number of attempts to retrieve the thrombus was limited to 5 passes by the occluded vessel. Neither IV heparin nor intra-arterial fibrinolytics were administered at any time during the procedure. Blood pressure was carefully monitored during anesthetic induction and during the procedure, with a minimal threshold set at $90 \mathrm{~mm} \mathrm{Hg}$ (mean arterial pressure). Hypotension was rapidly corrected if needed. Following any complications, extubation was planned at the end of the procedure and the patient was transferred to the intensive care unit.

Follow-up CT or MR imaging was performed 24 hours after the acute therapy to assess the extent of the infarction and/or hemorrhagic complications. If no hemorrhage was present, antiplatelet drugs were administered.

\section{Outcome Measures}

Successful recanalization, defined as TICI $2 \mathrm{~b}$ or 3 , was assessed at the end of the procedure in patients treated with thrombectomy. In patients treated with IVT, recanalization at 1 day was considered successful if follow-up MRA or angio-CT demonstrated complete visualization of the occluded artery, without residual stenosis of $>50 \%$.

"Symptomatic intracranial hemorrhage" (sICH) was defined as a documented hemorrhage on CT or MR imaging with a decline of $\geq 4$ points in the NIHSS score. Device-related complications were also reported. Clinical outcome was quantified by $\mathrm{mRS}$ 
and mortality at day 90 . Favorable outcome at 90 days was defined as an $\mathrm{mRS}$ score of $\leq 2$.

\section{Statistical Analysis}

Patients with a favorable or poor outcome were compared by using the Student $t$ or Mann-Whitney test for continuous variables and the $\chi^{2}$ or Fisher test for categoric variables. Bivariate logistic regressions were used to identify predictors of favorable outcome. Potential independent predictors $(P<.25$ in bivariate logistic regressions) were included in a multivariate logistic regression, built by stepwise procedure. Adjusted odds ratios and their $95 \%$ confidence intervals were calculated. The statistical significance threshold was set at 5\%. Statistical analyses were conducted by using SAS software 9.2 (SAS Institute, Cary, North Carolina).

\section{RESULTS}

\section{Population Data}

Overall, 41 patients with unclear-onset stroke in the anterior circulation (median age, 72 years; range, 17-89 years; female/ male ratio, 26:15) were included. Baseline clinical and radiologic features and main clinical outcomes at day 90 are presented in the Online Table.

The median time interval between last-seen-normal time and hospital admission was 490 minutes (interquartile range, 255641 minutes). The mean NIHSS score was $14.5 \pm 5.7$. The median time interval from FAT to MR imaging was 135 minutes (interquartile range, 109-158 minutes) and 517 minutes (interquartile range, 314-678 minutes) from last-seen-normal time. The median DWI ASPECTS was 8 (range, 5-10). Thirty-six patients $(88 \%)$ showed an arterial occlusion in the anterior circulation. Occlusion sites were the proximal MCA (M1) in 28 cases $(68.3 \%)$, the distal MCA (M2, M3) in 5 cases $(12.1 \%)$, and the anterior cerebral artery in 3 cases $(7.3 \%)$. The internal carotid artery was occluded in 14 patients (34.1\%), including 9 patients with tandem cervical ICA and intracranial occlusions and 5 carotid bifurcation occlusions.

\section{Stroke-Management Protocol}

Nineteen patients $(46.3 \%)$ underwent bridging therapy, and 21 patients (51.2\%), IVT alone. In these patients, the exclusion criteria for thrombectomy included the following: no occluded intracranial artery ( 5 patients), NIHSS score of $<8$ ( 4 patients), distal cerebral artery occlusion (5 patients), premorbid mRS of $>1$ ( 3 patients), fast recovery symptoms after IVT administration ( 1 patient), and delay beyond 6 hours after FAT ( 3 patients). The mean time from initial MR imaging to the start of IVT was $51 \pm$ 24 minutes. One patient with uncontrolled hypertension was treated by mechanical thrombectomy alone.

For the 20 patients treated by mechanical thrombectomy, the median number of passes with the thrombectomy device was 2 (range, 1-5). The thrombectomy procedure failed in 2 cases because of an inability to advance the microcatheter in the proximal ICA. Immediate successful recanalization (TICI $\geq 2 b$ ) was achieved in 15 patients (75\%), and TICI 3 was achieved in $40 \%$ $(8 / 20)$. The mean time from MR imaging to groin puncture was $81 \pm 38$ minutes. The median time from groin puncture to max- imum final TICI was 60 minutes (range, $21-248$ minutes). The mean time from FAT to recanalization was $295 \pm 75$ minutes and $686 \pm 196$ minutes from last-seen-normal-time.

\section{Outcome}

Four device-related complications occurred without neurologic deterioration or clinical sequelae, including 1 vessel perforation, 1 cervical ICA dissection, and 2 distal asymptomatic embolizations. Two $(4.9 \%)$ patients had sICH related to the acute therapy within the first 24 hours with a favorable outcome. Three (7.3\%) patients died during their hospital stay. Favorable outcome (mRS $\leq 2$ ) was observed in $25(61 \%)$ patients, including $14(70 \%)$ patients of the 20 treated by mechanical thrombectomy and $11(52 \%)$ of the 21 treated by IVT alone. A representative case is shown in Fig 1.

\section{Predictive Factors for Clinical Outcome}

Compared with patients treated by IVT alone, patients treated with bridging therapy showed a statistically significant difference in a number of parameters. Patients were younger $(P=.041)$ with a lower systolic blood pressure level at admission $(P=.017)$, a higher NIHSS score $(P=.007)$, and a lower ASPECTS $(P=.022)$. Their recanalization rate $(P=.023)$ was better (On-line Table).

The variables entered in the multivariate logistic regression analysis exploring predictive factors associated with a favorable outcome at day $90(\mathrm{mRS} \leq 2)$ were age, initial systolic blood pressure, initial blood glucose level, initial NIHSS score, IVT versus bridging therapy, and time interval from FAT to treatment initiation. In the final adjusted model, each 1-point decrease of the initial NIHSS score was independently associated with a favorable outcome at day 90 (OR, 1.43; 95\% CI, $1.13-1.82 ; P=.003)$ in patients with unclear-onset stroke and bridging therapy versus IV thrombolysis (OR, 37.92; 95\% CI, 2.43-591.35; $P=.009$ ) (Table).

\section{DISCUSSION}

Our study provided 2 important findings: 1) Reperfusion therapy based on DWI/FLAIR mismatch in patients with unclear-onset stroke seems to be efficient and safe, and 2) bridging therapy versus IVT alone is independently associated with favorable outcome at 3 months.

Our results are comparable with those of studies of IVT by using MR imaging variables (DWI/PWI, DWI/FLAIR mismatch) for patient selection, which showed favorable clinical outcome (40\%-56.3\% for mRS $0-2)$ and acceptable mortality $(0 \%-$ $10.3 \%)$ and $\mathrm{sICH}(0 \%-10.3 \%)$ rates. ${ }^{6-9}$ In contrast, CT-based thrombolysis in patients with wake-up stroke showed variable results. The only randomized controlled trial with thrombolytic treatment based on CT selection was stopped early because the rate of sICH was significantly higher in patients with wake-up stroke $(13.6 \%)$ than in the other patients with stroke $(4.0 \%){ }^{18}$ Barreto et al, ${ }^{4}$ by using noncontrast cranial CT, found, retrospectively, that 46 patients with intravenous thrombolysis and wake-up stroke had a significantly higher rate of favorable outcome $(28 \%$ versus $13 \% ; P=.006)$ but a higher mortality rate $(15 \%$ versus $0 \%)$ than 34 patients with wake-up stroke treated without thrombolysis.

In the literature, there are only a few reports of patients with an 

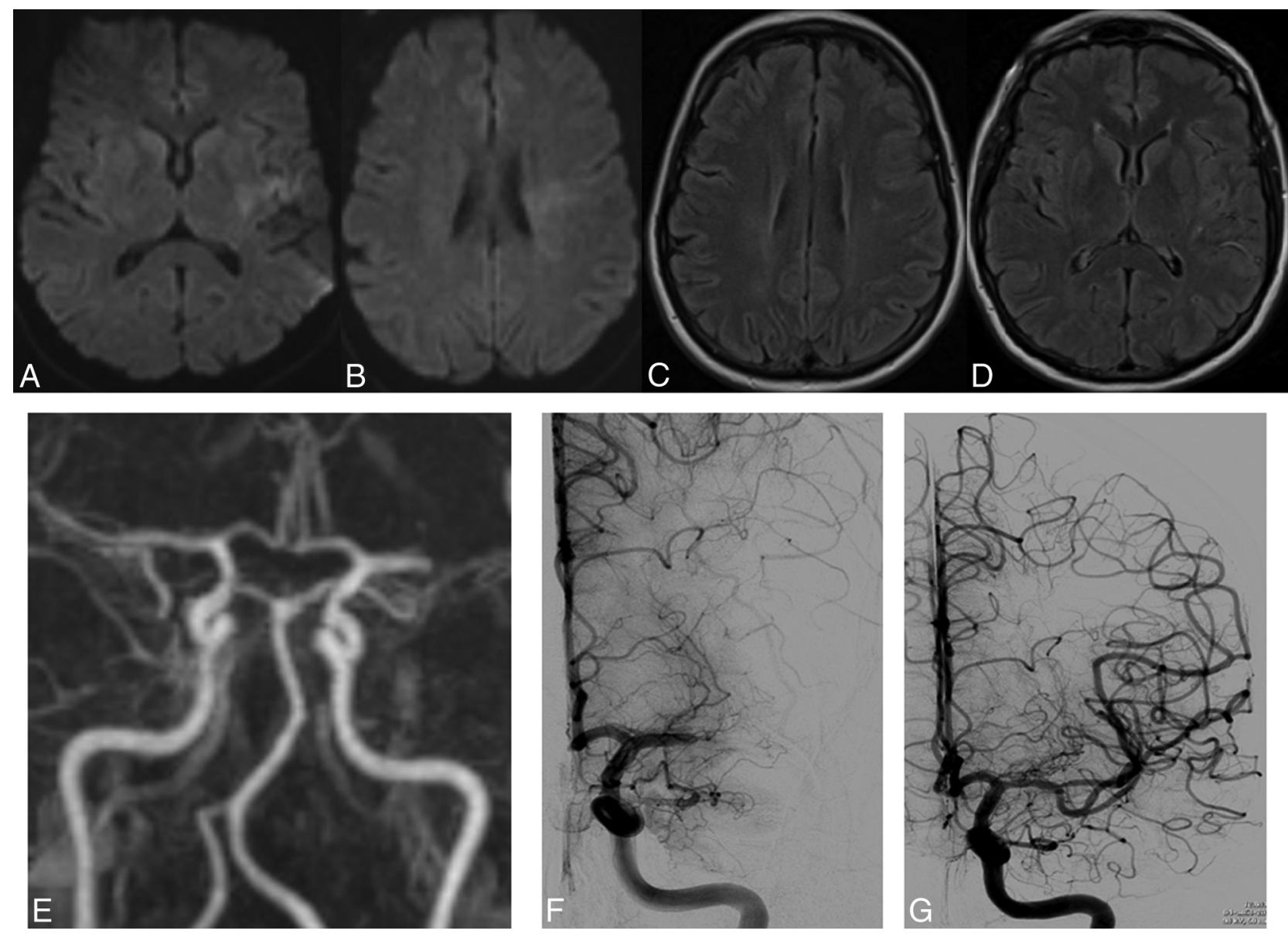

FIG 1. A 49-year-old woman presented with right hemiplegia and dysarthria (NIHSS score, 13). The patient arrived at the emergency department 72 minutes after symptom detection. MR imaging showed an acute ischemic lesion in the left MCA territory on DWI ( $A$ and $B$ ) without parenchymal signal changes on FLAIR ( $C$ and $D)$ and occlusion of the left MCA (M1 segment) with collateral blood flow in the distal cerebral artery territory on MRA $(E)$. Intravenous thrombolysis was started $(0.9 \mathrm{mg} / \mathrm{kg}) 140$ minutes after symptom detection. On DSA, the left MCA was still occluded on the Ml segment $(F)$ and was recanalized after mechanical thrombectomy $(\mathrm{TICI} 3)(G)$. Time from symptom detection to recanalization was 189 minutes (3 hours 9 minutes). The mRS score at 3 months was zero.

Predictive factors associated with favorable outcome at day 90 (mRS $\leq 2$ )

\begin{tabular}{|c|c|c|c|c|c|c|c|c|c|}
\hline \multirow[b]{2}{*}{ Variable } & \multirow[b]{3}{*}{$\begin{array}{l}\text { For each 1-point } \\
\text { decrease }\end{array}$} & \multicolumn{4}{|c|}{ Bivariate Logistic Regression } & \multicolumn{4}{|c|}{ Multivariate Logistic Regression } \\
\hline & & \multirow{2}{*}{$\begin{array}{l}\text { OR } \\
1.19\end{array}$} & \multicolumn{2}{|c|}{$95 \% \mathrm{Cl}$} & \multirow{2}{*}{$\begin{array}{c}\boldsymbol{P} \\
.017\end{array}$} & \multirow{2}{*}{$\frac{\text { OR }}{1.43}$} & \multicolumn{2}{|c|}{$95 \% \mathrm{Cl}$} & \multirow{2}{*}{$\begin{array}{c}\boldsymbol{P} \\
.003\end{array}$} \\
\hline Initial NIHSS & & & 1.03 & 1.37 & & & 1.13 & 1.82 & \\
\hline Treatment group & $\begin{array}{l}\text { Bridging versus } \\
\text { thrombolysis }\end{array}$ & 2.95 & 0.72 & 12.11 & .132 & 37.91 & 2.43 & 591.35 & .009 \\
\hline Blood glucose & $\begin{array}{l}\text { For each } 0.5-\mathrm{mmol} / \mathrm{L} \\
\text { increase }\end{array}$ & 0.82 & 0.66 & 1.02 & .081 & & & & \\
\hline Age & For each 5-year increase & 0.79 & 0.60 & 1.04 & .094 & & & & \\
\hline $\begin{array}{l}\text { Systolic blood } \\
\text { pressure }\end{array}$ & $\begin{array}{l}\text { For each } 10-\mathrm{mm} \\
\text { Hg increase }\end{array}$ & 0.80 & 0.59 & 1.09 & .160 & & & & \\
\hline $\begin{array}{l}\text { FAT-to-treatment } \\
\text { delay }\end{array}$ & $\begin{array}{l}\text { For each } 30 \text {-minute } \\
\text { increase }\end{array}$ & 0.81 & 0.50 & 1.30 & .377 & & & & \\
\hline
\end{tabular}

acute stroke of unknown time of onset who underwent multimodal reperfusion therapy or endovascular therapy. In the Reperfusion Therapy in Unclear-Onset Stroke Based on MRI Evaluation trial, a prospective study using MR imaging criteria in 83 patients with an unknown time of onset, ${ }^{19}$ more than two-thirds of patients received endovascular treatment and approximately $10 \%$ received IVT alone. Favorable outcome (mRS $0-2$ ) was achieved in $44.6 \%$, and sICH was seen in $3.6 \%$. Natarajan et $\mathrm{al}^{11}$ performed a retrospective review of 30 patients with significant salvageable brain tissue identified on CT perfusion who underwent endovascular recanalization (intra-arterial thrombolysis, mechanical thrombectomy, or angioplasty) $\geq 8$ hours after lastseen-normal time, including those with wake-up stroke; partial/ complete recanalization was achieved in $66.7 \%$ of patients, with $20 \%$ presenting with mRS $0-2$ at 3 months. Overall mortality was $33.3 \%$, and sICH was $10 \%$. 
Recently, Stampfl et al ${ }^{12}$ retrospectively analyzed clinical and angiographic data in 19 patients with wake-up stroke and diffusion/perfusion mismatch on MR or CT imaging treated with stent-retriever devices. Despite successful and rapid recanalization ( $94.7 \%$ of TICI $\geq 2$ ), clinical outcome remained poor $(10.5 \%$ of mRS $0-2 ; 36.8 \%$ died), and sICH occurred in $21.1 \%$ of patients. The authors underlined the difficulty of patient selection for endovascular therapy. Despite a lower recanalization rate, our results are better than those previously published for multimodal reperfusion therapy ${ }^{11,19}$ or endovascular treatment ${ }^{12}$ in terms of the frequency of good outcome (61\% versus $10.5 \%-44.6 \%)$, mortality (7.3\% versus $33.3 \%-36.8 \%)$, and sICH (4.9\% versus $3.6 \%-21.1 \%)$ rates.

Recently 3 randomized studies ${ }^{13-17}$ reported the superiority of rapid thrombectomy, compared with IVT alone, in patients with acute ischemic stroke with a proximal intracranial occlusion and improving reperfusion (66\%-100\% for intervention versus $31.2 \%-37 \%$ for controls) and functional outcome at 90 days (32.6\%-71\% for intervention versus $19.1 \%-40 \%$ for controls). There were no significant differences in mortality $(9 \%-18.9 \%$ for intervention versus $12 \%-20 \%$ for controls) or the occurrence of symptomatic intracerebral hemorrhage $(0 \%-7.7 \%$ for intervention versus $1.9 \%-6.4 \%$ for controls).

In our study, patients treated with bridging therapy had more favorable outcome compared with patients treated with IVT alone. These results were shown despite the conditions of patients treated with bridging being more severe (worst NIHSS and ASPECTS at admission). Indeed, after adjusting for the initial NIHSS score, bridging therapy versus IVT alone was independently associated with favorable outcome at 3 months in patients with unclear-onset stroke. Even if there is a statistically significant difference between these 2 groups of patients, our results suggest that IVT followed by endovascular therapy combines the advantages of a rapid start of treatment with IVT $^{2}$ and a greater likelihood of early recanalization.

Recently, several trials emphasized the importance of patient selection for reperfusion therapy, with the tissue clock shown by multiparametric MR imaging techniques. ${ }^{6-9,12}$ We selected, in our study, patients using DWI/FLAIR mismatch and clinical-diffusion mismatch among MR imaging criteria. As reported by Thomalla et $\mathrm{al}^{3}{ }^{3}$ a patient with an acute ischemic lesion detected with DWI but not with FLAIR imaging is likely to be within 4.5 hours of symptom onset with high specificity (78\%; 95\% CI, $72 \%-84 \%)$ and high positive predict value (83\%; 95\% CI, 79\%$88 \%)$. Moreover, clinical-diffusion mismatch, which predicts the presence of PWI-DWI mismatch, may be associated with neurologic improvement in patients treated with IVT as reported by Terasawa et al. ${ }^{20}$ Indeed, a number of studies have provided support for penumbral-imaging selection, by using the perfusiondiffusion mismatch criteria for the treatment of acute ischemic stroke $e^{7-9,12}$ because salvage of the ischemic penumbra has formed the theoretic basis of recanalization therapies. This criterion may be particularly helpful in late time windows, when the proportion of patients with penumbral tissue steadily decreases. Last, the results of Diffusion and Perfusion Imaging Evaluation for Understanding Stroke Evolution Study 2 demonstrated that reperfusion was associated with increased good functional outcome at day 9 in a defined target mismatch profile. ${ }^{21}$

Obvious limitations of our monocentric observational study were the small number of patients, a retrospective analysis of our prospective dataset performed, and no control group. Using predefined imaging criteria, several ongoing prospective clinical trials are testing the safety and efficiency of thrombolytic treatment in patients with stroke with an unknown time of onset. ${ }^{22}$

\section{CONCLUSIONS}

Our preliminary results suggest that MR imaging-based reperfusion therapy can safely and efficiently be applied to patients with acute stroke with an unknown time of onset (based on DWI/ FLAIR mismatch). Moreover, this study underlines the fact that IVT combined with endovascular mechanical thrombectomy seems to be associated with favorable clinical outcome when patients are carefully selected. Nevertheless, multicenter randomized trials are required to confirm these results and to determine the optimal multimodal MR imaging criteria for patient selection and the optimal treatment strategies.

Disclosures: Alain Bonafé-UNRELATED: Consultancy: Covidien (consultant for ev3), Stryker; Grants/Grants Pending: Covidien.* Vincent Costalat-Consultancy: Balt, Codman Neuro-DePuy Synthes, Stryker, MicroVention; Payment for Lectures (including service on Speakers Bureaus): Stryker, Balt; Payment for Development of Educational Presentations: Covidien, Stryker. *Money paid to the institution.

\section{REFERENCES}

1. Fink JN, Kumar S, Horkan C, et al. The stroke patient who woke up: clinical and radiological features, including diffusion and perfusion MRI. Stroke 2002;33:988-93 CrossRef Medline

2. Hacke W, Kaste M, Bluhmki E, et al; ECASS Investigators. Thrombolysis with alteplase 3 to 4.5 hours after acute ischemic stroke. N Engl J Med 2008;359:1317-29 CrossRef Medline

3. Thomalla G, Cheng B, Ebinger M, et al; STIR and VISTA Imaging Investigators. DWI-FLAIR mismatch for the identification of patients with acute ischaemic stroke within $4.5 \mathrm{~h}$ of symptom onset (PRE-FLAIR): a multicentre observational study. Lancet Neurol 2011;10:978-86 CrossRef Medline

4. Barreto AD, Martin-Schild S, Hallevi H, et al. Thrombolytic therapy for patients who wake-up with stroke. Stroke 2009;40:827-32 CrossRef Medline

5. Cortijo E, Garcia-Bermejo P, Calleja AI, et al. Intravenous thrombolysis in ischemic stroke with unknown onset using CT perfusion. Acta Neurol Scand 2014;129:178-83 CrossRef Medline

6. Aoki J, Kimura K, Iguchi Y, et al. Intravenous thrombolysis based on diffusion-weighted imaging and fluid-attenuated inversion recovery mismatch in acute stroke patients with unknown onset time. Cerebrovasc Dis 2011;31:431-41 CrossRef Medline

7. Breuer L, Schellinger PD, Huttner HB, et al. Feasibility and safety of magnetic resonance imaging-based thrombolysis in patients with stroke on awakening: initial single-centre experience. Int J Stroke 2010;5:68-73 CrossRef Medline

8. Cho AH, Sohn SI, Han MK, et al. Safety and efficacy of MRI-based thrombolysis in unclear-onset stroke: a preliminary report. Cerebrovasc Dis 2008;25:572-79 CrossRef Medline

9. Kim JT, Park MS, Nam TS, et al. Thrombolysis as a factor associated with favorable outcomes in patients with unclear-onset stroke. Eur J Neurol 2011;18:988-94 CrossRef Medline

10. Kuruvilla A, Norris GM, Xavier AR. Acute endovascular recanalization therapy in wake-up stroke. J Neurol Sci 2011;300:148-50 CrossRef Medline

11. Natarajan SK, Snyder KV, Siddiqui AH, et al. Safety and effective- 
ness of endovascular therapy after 8 hours of acute ischemic stroke onset and wake-up strokes. Stroke 2009;40:3269-74 CrossRef Medline

12. Stampfl S, Ringleb PA, Haehnel S, et al. Recanalization with stentretriever devices in patients with wake-up stroke. AJNR Am J Neuroradiol 2013;34:1040 - 43 CrossRef Medline

13. Berkhemer OA, Fransen PSS, Beumer D, et al. A randomized trial of intraarterial treatment for acute ischemic stroke. N Engl J Med 2015; 372:11-20 CrossRef Medline

14. Campbell BCV, Mitchell PJ, Kleinig TJ, at al; EXTEND-IA Investigators. Endovascular therapy for ischemic stroke with perfusion-imaging selection. $N$ Engl J Med 2015;372:1009-18 CrossRef Medline

15. Goyal M, Demchuk AM, Menon BK, et al; ESCAPE Trial Investigators. Randomized assessment of rapid endovascular treatment of ischemic stroke. $N$ Engl J Med 2015;372:1019-30 CrossRef Medline

16. Jovin TG, Chamorro A, Cobo E, et al; REVASCAT Trial Investigators. Thrombectomy within $\mathbf{8}$ hours after symptom onset in ischemic stroke. N Engl J Med 2015;372:2296-306 CrossRef Medline
17. Saver JL, Goyal M, Bonafe A, et al; SWIFT PRIME Investigators. Stent-retriever thrombectomy after intravenous t-PA vs. t-PA alone in stroke. N Engl J Med 2015;372:2285-95 CrossRef Medline

18. Adams HP Jr, Leira EC, Torner JC, et al; AbESTT-II Investigators. Treating patients with 'wake-up' stroke: the experience of the AbESTT-II trial. Stroke 2008;39:3277-82 CrossRef Medline

19. Kang DW, Sohn SI, Hong KS, et al. Reperfusion therapy in unclearonset stroke based on MRI evaluation (RESTORE): a prospective multicenter study. Stroke 2012;43:3278-83 CrossRef Medline

20. Terasawa Y, Kimura K, Iguchi Y, et al. Could clinical diffusion-mismatch determined using DWI ASPECTS predict neurological improvement after thrombolysis before $3 \mathrm{~h}$ after acute stroke? J Neurol Neurosurg Psychiatry 2010;81:864-68 CrossRef Medline

21. Lansberg MG, Straka M, Kemp S, et al; DEFUSE 2 study investigators. MRI profile and response to endovascular reperfusion after stroke (DEFUSE 2): a prospective cohort study. Lancet Neurol 2012;11: 860-67 CrossRef Medline

22. Kang DW, Kwon JY, Kwon SU, et al. Wake-up or unclear-onset strokes: are they waking up to the world of thrombolysis therapy? Int J Stroke 2012;7:311-20 CrossRef Medline 